\title{
Molecular imaging in oncology: the acceptance of PET/CT and the emergence of MR/PET imaging
}

\author{
Christiaan Schiepers • Magnus Dahlbom
}

Received: 25 November 2010 / Accepted: 2 December 2010 /Published online: 21 December 2010

(C) The Author(s) 2010. This article is published with open access at Springerlink.com

\begin{abstract}
In the last decade, PET-only systems have been phased out and replaced with PET-CT systems. This merger of a functional and anatomical imaging modality turned out to be extremely useful in clinical practice. Currently, PET$\mathrm{CT}$ is a major diagnostic tool in oncology. At the dawn of the merger of MRI and PET, another breakthrough in clinical imaging is expected. The combination of these imaging modalities is challenging, but has particular features such as imaging biological processes at the same time in specific body locations.
\end{abstract}

Keywords CT - Computed tomography PET—Positron Emission Tomography $\cdot$ MRI-Magnetic Resonance Imaging · MRS - Magnetic Resonance Spectroscopy . Staging of Cancer - Therapy Monitoring .

FDG- ${ }^{18}$ F Fluoro-deoxy-glucose

\section{Introduction}

In oncology, detection of tumor, staging of disease, and monitoring of therapy are important for patient management and prognosis [1]. Cancer patients undergo a number of different imaging studies throughout the course of their disease. Various imaging investigations are available and the resulting images are reviewed on different viewing stations. The gathered information is synthesized and integrated by the interpreting physician(s). The multitude

C. Schiepers $(\square) \cdot$ M. Dahlbom

Molecular \& Medical Pharmacology,

David Geffen School of Medicine at UCLA,

University of California,

200 Medical Plaza, Suite B-114,

Los Angeles, CA 90095-7370, USA

e-mail: cschiepers@mednet.ucla.edu of images need to be correlated, and can be co-registered or aligned retrospectively and fused in computer memory. Post hoc image fusion, based on tomograms acquired at different institutions, on separate days, using varying equipment and protocols, is a tedious and time-consuming task. Moreover, well-defined and reproducible landmarks are necessary to provide the coordinate system in which the images can be aligned, scaled and registered. Since patient positioning varies widely between PET and CT, e.g. arms up or down, different patient and organ axes, the post hoc fusion technique is prone to mis-alignment, inconsistencies and errors. Also, there may be problems due to patient movement as well as motion of internal organs. Changes related to breathing and organ movement such as a beating heart, are inevitable, and cannot be controlled when a patient is imaged on different machines and at different times, even when care is taken to ensure that the body position of the patient is the same (e.g. with external lasers in 3D). Other movements such as bowel peristalsis may be minimized with medication. Brown adipose tissue uptake may be aborted with sympathetic nervous system receptor blocking agents.

In the last decade, this journal has reported on the developments of imaging in oncology, from the progress of PET-only [2] to combining PET and CT [3] and PET and MRI [4]. Reviews have been published on some of the achievements of the dual imaging modality PET/CT, a powerful, routinely and frequently applied molecular imaging tool for diagnosis, staging and therapy monitoring in oncology [5-8].

\section{PET-CT imaging}

For an oncology study the most often used radiopharmaceutical is ${ }^{18} \mathrm{~F}-\mathrm{FDG}$ [9]. FDG mimics the glucose utilization, 
which is usually deranged in cancer. Typically, 200$600 \mathrm{MBq}$ of ${ }^{18} \mathrm{~F}-\mathrm{FDG}$ is administered to the patient, followed by a $1 \mathrm{~h}$ uptake interval. During this uptake interval, oral contrast medium may be administered, e.g. at 5, 30 , and 55 min relative to the FDG injection, for improved detection of abdominal and pelvic abnormalities by CT. After voiding, the patient is positioned in the PET/CT system. The photon emissions from the ${ }^{18} \mathrm{~F}$ label do not interfere with the CT detectors, which have lower sensitivity for the high-energy photons from the annihilation. In addition, the photon flux from ${ }^{18} \mathrm{~F}$-FDG is a power of 4 lower than from the X-ray tube.

A scout view is obtained and the imaging field defined. Subsequently, a helical CT dataset is acquired without and/ or with intravenous contrast medium. The complete CT data acquisition takes $0.5-1.5 \mathrm{~min}$, depending on the axial field of view and type of CT system incorporated into the $\mathrm{PET} / \mathrm{CT}$ machine. The patient bed is then translated axially into the PET field-of-view and PET data [10] acquired over the same axial range as the CT. The duration of the PET data acquisition is $5-25 \mathrm{~min}$ dependent on the axial fieldof-view, the type of detectors and mode of acquisition (2D vs $3 \mathrm{D})$. The CT data are used for attenuation correction, omitting the lengthy transmission with radioactive sources which was used previously [3]. If an enhanced CT study is requested or deemed appropriate, the additional $\mathrm{CT}$ acquisition is performed after the standard PET-CT study is completed, to ensure correct attenuation correction [3]. Intravenous contrast medium (usually about $300 \mathrm{mg} / \mathrm{ml}$ iodine) at a rate of $1-2 \mathrm{ml} / \mathrm{sec}$ is administered up to $125 \mathrm{ml}$. Current multidetector CT systems are sufficiently fast to permit multi-phase imaging. An enhanced CT following PET/ CT is generally limited to a specific area of the body, e.g. chest, liver or pelvis, in order to reduce the total radiation dose to the patient. If a dedicated head \& neck CT examination is required, the intravenous dose of contrast agent can be split in two, $80 \mathrm{ml}$ for the body followed by $45 \mathrm{ml}$ for the H\&N .

The absorbed radiation dose from some whole body CT examinations is significant ( $7-14 \mathrm{mSv})$, and an additional dedicated $\mathrm{CT}$ increases the absorbed radiation dose.

The combined CT-PET-CT acquisition generally takes less than half an hour.

The contribution of breathing is less important for the abdomen than for the chest [11]. The artifact caused by motion of the liver during $\mathrm{CT}$ acquisition, does not pose a real clinical problem in staging primary hepatic cancer or extent of liver metastases [12]. Non-attenuation corrected tomograms and projection images are always available to check for possible artifacts induced by mis-registered PETCT slices. Metal implants or contrast material may also induce artifacts. Therefore, non-attenuation corrected images are routinely interpreted to check for and eliminate possible imaging artifacts. Modern reconstruction algo- rithms are less sensitive to metal artifacts, dental implants, oral and intravenous contrast agents [13, 14].

During the last decade, PET-only systems have disappeared from the clinical armamentarium, and have largely been replaced with PET-CT equipment. From a patient point of view, it is convenient only to have to make a single visit to the imaging suite for diagnostic work-up. Several reviews on the achievements are available in the literature [15-18].

In 2003, Blomqvist and Torkzad wrote in JAMA [19] that: 'the dual-imaging modality PET/CT is relatively new', and is used mostly: 'in patients with lung cancer'. As of 2010, the application of this type of dual modality diagnostic imaging is the standard in oncology and the current state-ofthe-art in staging of disease and monitoring of treatment.

Many studies have been reported and reviews published for common and less common cancers [16, 20-23]; others were focused on lung $[17,24]$, breast [25-29], colorectal [30-32], recurrent rectal [33], head \& neck [34-36], ovarian [22], uterine [37] and cervical [38, 39], nasopharyngeal [40] and thyroid cancer [41, 42], melanoma [4345], as well as lympho-proliferative disorders [46-48], carcinoma of unknown primary (CUP) [49], and primary muscular-skeletal tumors [8].

Several publications deal with false positive results [50], artifacts and pitfalls [51], and with problems about SUV $[52,53]$, the standardized uptake value, a parameter that is used for semi-quantitative purposes, especially for longitudinal and serial comparisons [54]. Bowel preparation was reported by Blake et al. [55].

The role of FDG in therapy monitoring was reviewed in 2005 [6] and 2009 [56].

The contribution of PET/CT in radiation therapy planning has been established for the common cancers [57-59], and head \& neck [60].

The latest addition to clinical PET/CT imaging is Timeof-Flight (TOF), where the time differences between the detection of the annihilation photons is taken into account. Using this information in the image reconstruction, it can be shown that a gain in image signal-to-noise ratio can be achieved, in particular for large objects [61]. This technique is not new, was introduced a few decades ago [62] but lacked the proper detection material and electronics to produce the anticipated improvement in image quality. Using TOF indirectly improves the spatial resolution since less spatial filtering is necessary to maintain the image signal-to-noise $(\mathrm{S} / \mathrm{N})$ ratio. However, TOF requires fast scintillation detectors and additional fast and optimized electronics, which increases the cost. The intrinsic FWHM of a modern PET/CT scanner such as the Siemens ${ }^{\circledR} \mathrm{mCT}^{\circledR}$ (Forchheim, Germany) with TOF and ultra-HD PET, improves the spatial resolution from 4 to $2 \mathrm{~mm}$.

In Fig. 1, images are shown of a diagnostic study with conventional PET/CT and TOF-PET/CT. 
a
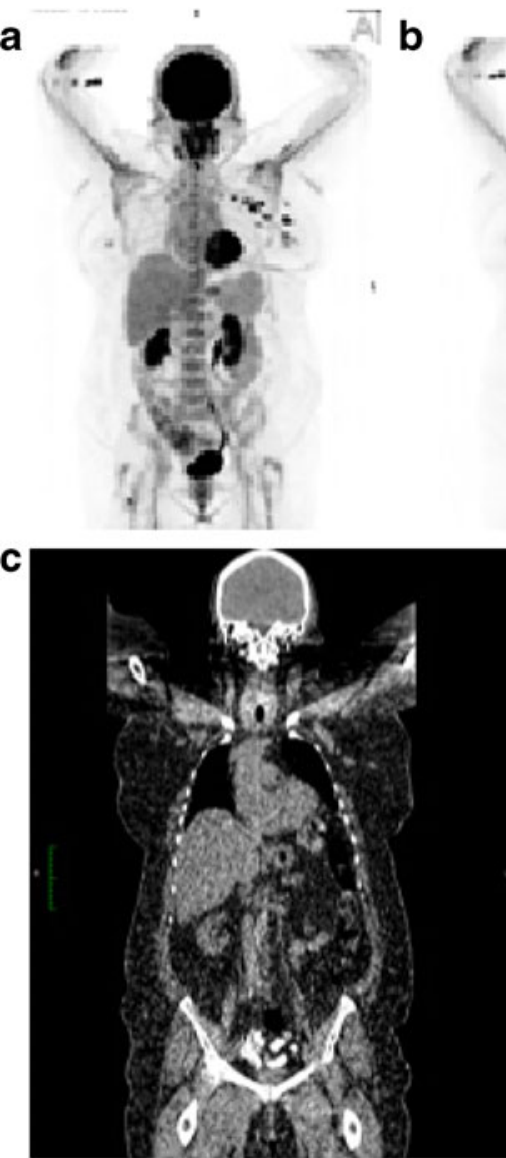

d
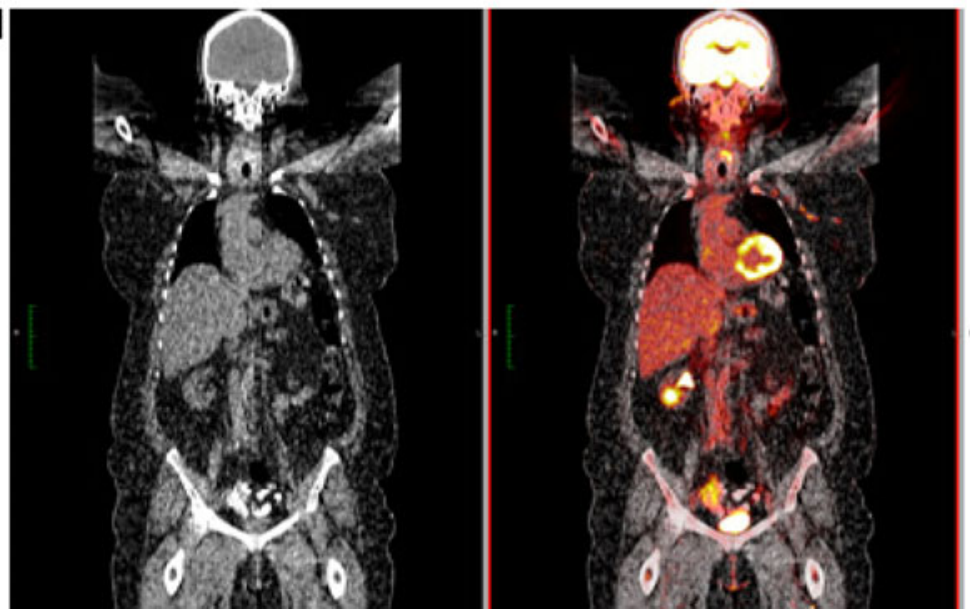
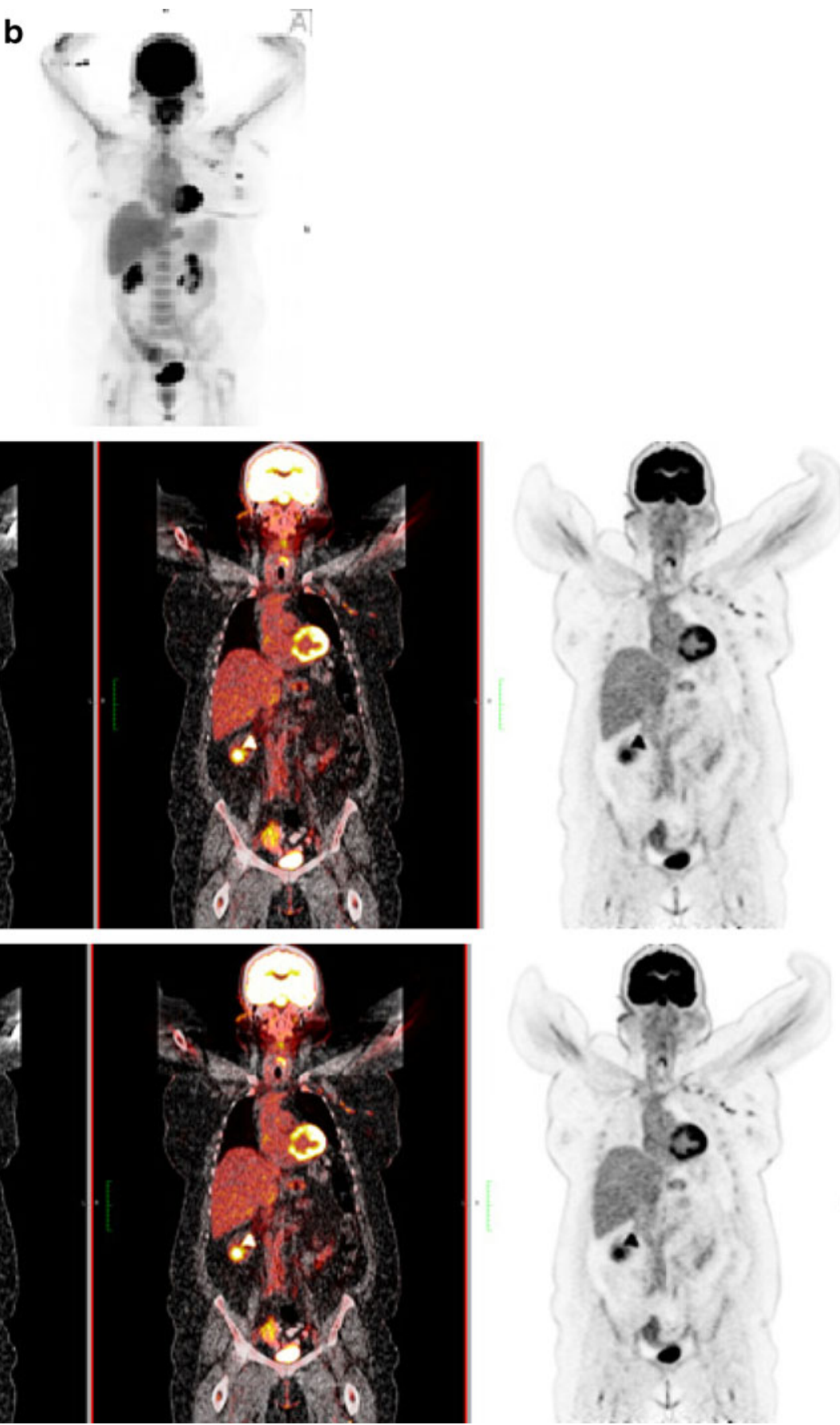

Fig. 1 Maximum projection image in the anterior view for TOF-PET (a) and PET (b) of a woman with breast cancer. Note the difference in contrast and intensity of the lesions in the left breast and axilla. Coronal slices for CT (left panel) and PET (right panel), and the fused PET-CT (middle) are given for TOF-PET (c) and PET (d). The difference in contrast can still be appreciated. TOF contributes most in

\section{MR-PET imaging}

Combining anatomical MR imaging with the molecular PET, potentially offers the advantages that are seen in PET/ CT. In addition, the advent of MR spectroscopy (MRS), obese patients, who have higher scatter of the emitted photons. Preservation of the $\mathrm{S} / \mathrm{N}$ ratio and improved spatial resolution of TOF enhances lesion contrast. Patient weight was $120.2 \mathrm{~kg}$; administered activity $606 \mathrm{MBq}$ of ${ }^{18} \mathrm{~F}$-FDG; total acquisition time of PET/CT was $21 \mathrm{~min}$

another molecular imaging technique, might be fused as well [3].

Post hoc or software based image fusion of MR and PET images has the same disadvantages as PET/CT. For PET/ $\mathrm{CT}$, the solution turned out to be 'mechanical' or 'in-line' 
fusion. This means that the acquisitions of CT and PET are separated in space and in time, and are not simultaneous. The bed for image acquisition, however, is the same for $\mathrm{CT}$ and PET; the patient is in the same position on this bed, whereas fusion happens later in computer memory. The main reason for non-simultaneous acquisition is the lack of detectors with 'reasonable' efficiency for both the 60$120 \mathrm{keV}$ photons from $\mathrm{CT}$ and the $511 \mathrm{keV}$ annihilation photons from PET [3]. Since the detectors for MR and PET imaging are totally different, another type of gantry is necessary to combine these two modalities. MR imaging takes much longer than multi-detector $\mathrm{CT}$ and the acquisition times for MR and PET become similar. Thus, the 'simultaneous' acquisition option for MR and PET appears extremely appealing (Fig. 2). The 'sequential' option (used in PET/CT) would lead to much longer acquisition times for MR/PET, i.e. time that the patient has to be inside the scanners (Fig. 3). In other words, patient movement and motion artifacts are able to cause mis-registration and disrupt the final image fusion quality.

A paramount advantage of the simultaneous acquisition option is that we are interrogating and measuring the tissues of the patient at exactly the same time. Thus, image registration becomes trivial [63].

MR has no ionizing radiation, so the combined MR/PET and MRS absorbed radiation dose to the patient is solely due to the administered radiopharmaceutical. This is much more favorable than $\mathrm{CT}$, which has a relatively high radiation burden, especially with the current deep inspiration, multi-phase with and without contrast medium, and shallow breathing protocols.

There are a number of challenges in combining PET and MRI. The PMTs (photo-multiplier tubes) used in conven-

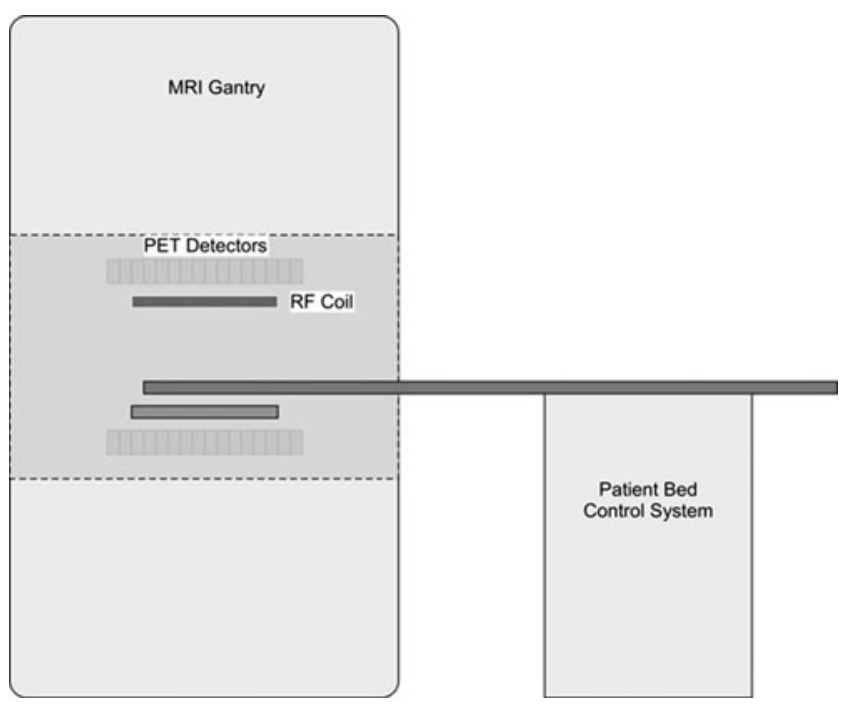

Fig. 2 Simultaneous acquisition of MR and PET data. The RF (radiofrequency) coil is located inside the ring of PET detectors tional PET detectors are highly sensitive to magnetic fields and cannot be used near the MR magnet. Solid-state detectors are less sensitive to magnetic fields and have been shown to operate relatively undisturbed in strong magnetic fields. These detectors have to be constructed without any ferromagnetic materials that would otherwise produce heterogeneity within the magnetic field. These challenges have therefore resulted in different approaches for combined MR/PET systems [64].

\section{Simultaneous acquisition}

In order to simultaneously perform PET and MR imaging, the PET detectors have to be able to operate inside the magnetic field without interfering with the MR imaging. These types of detectors have successfully been constructed and are typically based on silicon avalanche photo-detectors (APDs) [64]. Several animal systems based on this technology have been constructed and Siemens ${ }^{\circledR}$ introduced a PET insert for one of their MR systems in 2007. This system has a relatively long axial FOV $19.25 \mathrm{~cm}$, but because of the small detector diameter of $35.5 \mathrm{~cm}$ it is limited to brain imaging [65]. A recent clinical study comparing PET/CT and MR/PET imaging showed that the image quality was similar for both systems [66]. MR/PET offers additional advantages such as higher tissue contrast, sophisticated MR imaging techniques such as perfusion and diffusion imaging [67], and MR spectroscopy, without adding extra radiation to the patient. It is very likely that this technology will be extended to clinical whole body imaging in the very near future [4].

\section{Sequential acquisition}

In the Philips' ${ }^{\circledR}$ (Eindhoven, Netherlands) MR/PET system, a different system design is used. The conventional PET and MRI gantries are placed at some distance from each other in order to minimize interference between the two systems. The two devices use a common patient bed, which can be moved into either imaging gantry. This solution does not require special PET detectors, although additional magnetic shielding is placed around the PET gantry to minimize the effect on the PMTs. This solution has the drawback of not allowing simultaneous PET and MR imaging, which prevents imaging of physiological and biochemical processes at the same moment in time by the two modalities. Since the PET and MRI data sets are acquired sequentially and since MR imaging usually takes much longer than $\mathrm{CT}$, the imaging sessions are substantially longer compared to corresponding $\mathrm{PET} / \mathrm{CT}$ imaging procedures. 


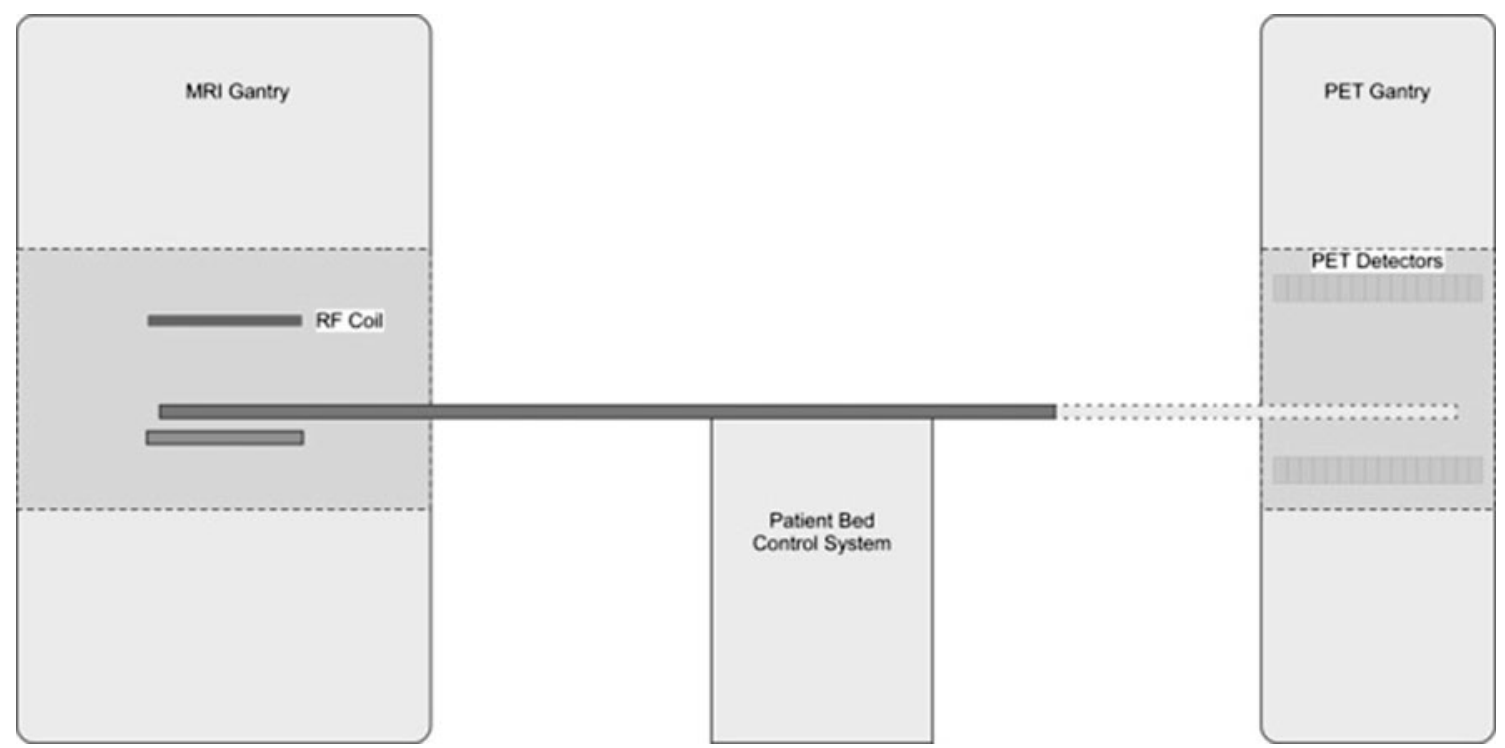

Fig. 3 Sequential acquisition, i.e. both in space and in time, of MR and PET data. The two gantries are standard and in different locations. Additional shielding against the magnetic field is necessary for the PET electronics

Topics for more research

Current limitations of MR are the thick slices (5-7 mm in zplane), whereas PET is already at $2-4 \mathrm{~mm}$, especially with the TOF electronics.

Another challenge for a combined MR/PET system is the attenuation correction [68]. Since a set of CT images represent a map of attenuation coefficients, these images can relatively easily be converted to attenuation maps at $511 \mathrm{keV}$ using a scaling technique.

However, the pixel values in an MR image represent completely different physical properties (i.e., proton density, relaxation times, etc), and the conversion of these pixel values to attenuation coefficients at $511 \mathrm{keV}$ is far from straightforward. Since the attenuation coefficients in soft tissue are relatively uniform at $511 \mathrm{keV}$, most visualized tissue types can be assigned a population average. However, tissues of significantly lower density such as lung may require built-in intelligence in the processing software to assign the correct attenuation values. An additional complication is that not all tissue types are visualized in MRI (e.g. bone). This may require the use of deformable anatomical atlases to generate the correct attenuation coefficients.

Further complications in using the MR images for attenuation correction is that many of the objects used to form the MR images (e.g., coils) are not visualized in the MR images, but will attenuate the $511 \mathrm{keV}$ photons. Attenuation by these objects will therefore have to be measured, and their locations during the MR/PET data acquisition have to be determined. The conversion of MR images to attenuation maps is a complex problem and is an area of active research. A number of different approaches are currently being investigated and developed [4, 65].

\section{Summary}

Our experience shows that the field of combined and correlative imaging is changing, and the radiologist or nuclear medicine specialist must be eager to participate in this evolving field of molecular imaging. For oncological applications, the importance of having a detailed anatomical framework that permits accurate interpretation of functional images cannot be over-emphasized. The requirements for such a reference framework will become increasingly important with the development of more specific tracers such as amino-acids, precursor, transporter, receptor and gene-imagers [69].

Here, we alluded to the acceptance of PET/CT imaging as the standard in diagnostic imaging for oncology. Patient preparation is similar for a PET/CT compared to a routine CT or PET study. For patients, the dual imaging session is beneficial by saving time. For imaging specialists, the combined equipment enhances patient throughput, and technological innovations lead to superior image quality. Image fusion has improved lesion localization, and increased accuracy in staging of disease.

It is expected that MR/PET will follow a similar stellar development. The simultaneous acquisition of data in this dual imaging setting will undoubtedly benefit image registration and reduce artifacts, and thus improve accuracy. Correction for photon attenuation of the PET images is not as straightforward as it was with PET/CT but several 
solutions are possible. The combination with techniques such as diffusion and perfusion imaging, as well as molecular imaging with MR spectroscopy will advance the multimodality-imaging field to even higher levels and broader applications.

Open Access This article is distributed under the terms of the Creative Commons Attribution Noncommercial License which permits any noncommercial use, distribution, and reproduction in any medium, provided the original author(s) and source are credited.

\section{References}

1. Jemal A, Siegel R, Ward E et al (2009) Cancer statistics, 2009. CA Cancer J Clin 59:225-249

2. Schiepers C, Hoh CK (1998) Positron emission tomography as a diagnostic tool in oncology. Eur Radiol 8:1481-1494

3. Townsend DW, Cherry SR (2001) Combining anatomy and function: the path to true image fusion. Eur Radiol 11:1968-1974

4. Pichler BJ, Kolb A, Nagele T et al (2010) PET/MRI: paving the way for the next generation of clinical multimodality imaging applications. J Nucl Med 51:333-336

5. Siegel BA, Dehdashti F (2005) Oncologic PET/CT: current status and controversies. Eur Radiol 15(Suppl 4):D127-132

6. Denecke T, Rau B, Hoffmann KT et al (2005) Comparison of CT, MRI and FDG-PET in response prediction of patients with locally advanced rectal cancer after multimodal preoperative therapy: is there a benefit in using functional imaging? Eur Radiol 15:1658-1666

7. Schmidt GP, Haug AR, Schoenberg SO et al (2006) Whole-body MRI and PET-CT in the management of cancer patients. Eur Radiol 16:1216-1225

8. Lakkaraju A, Patel CN, Bradley KM et al (2010) PET/CT in primary musculoskeletal tumours: a step forward. Eur Radiol 20:2959-2972

9. Gambhir SS, Czernin J, Schwimmer J et al (2001) A tabulated summary of the FDG PET literature. J Nucl Med 42:1S-93S

10. Dahlbom M, Hoffman EJ, Hoh CK et al (1992) Whole-body positron emission tomography. 1. Methods and performance characteristics. J Nucl Med 33:1191-1199

11. Goerres GW, Burger C, Schwitter MW et al (2003) PET/CT of the abdomen: optimizing the patient breathing pattern. Eur Radiol 13:734-739

12. de Juan R, Seifert B, Berthold T et al (2004) Clinical evaluation of a breathing protocol for PET/CT. Eur Radiol 14:1118-1123

13. Kinahan PE, Hasegawa BH, Beyer T (2003) X-ray-based attenuation correction for positron emission tomography/computed tomography scanners. Semin Nucl Med 33:166-179

14. Kamel EM, Burger C, Buck A et al (2003) Impact of metallic dental implants on CT-based attenuation correction in a combined PET/CT scanner. Eur Radiol 13:724-728

15. von Schulthess GK, Steinert HC, Hany TF (2006) Integrated PET/ CT: current applications and future directions. Radiology 238:405-422

16. Blodgett TM, Meltzer CC, Townsend DW (2007) PET/CT: form and function. Radiology 242:360-385

17. Freudenberg LS, Rosenbaum SJ, Beyer T et al (2010) PET versus $\mathrm{PET} / \mathrm{CT}$ dual-modality imaging in evaluation of lung cancer. Thorac Surg Clin 20:25-30

18. Ell PJ (2006) The contribution of PET/CT to improved patient management. Br J Radiol 79:32-36
19. Blomqvist L, Torkzad MR (2003) Whole-body imaging with MRI or PET/CT: the future for single-modality imaging in oncology? JAMA 290:3248-3249

20. Patil VV, Wang ZJ, Sollitto RA et al (2009) 18F-FDG PET/CT of transitional cell carcinoma. AJR Am J Roentgenol 193:W497-504

21. Podoloff DA, Macapinlac HA (2007) PET and PET/CT in management of the lymphomas. Radiol Clin North Am 45:689696, vii

22. Prakash P, Cronin CG, Blake MA (2010) Role of PET/CT in ovarian cancer. AJR Am J Roentgenol 194:W464-470

23. Wahl RL (2004) Why nearly all PET of abdominal and pelvic cancers will be performed as PET/CT. J Nucl Med 45(Suppl 1):82S-95S

24. Lardinois D, Weder W, Hany TF et al (2003) Staging of nonsmall-cell lung cancer with integrated positron-emission tomography and computed tomography. $N$ Engl J Med 348:2500-2507

25. Goerres GW, Michel SC, Fehr MK et al (2003) Follow-up of women with breast cancer: comparison between MRI and FDG PET. Eur Radiol 13:1635-1644

26. Fueger BJ, Weber WA, Quon A et al (2005) Performance of 2deoxy-2-[F-18]fluoro-D-glucose positron emission tomography and integrated PET/CT in restaged breast cancer patients. Mol Imaging Biol 7:369-376

27. Radan L, Ben-Haim S, Bar-Shalom R et al (2006) The role of FDG-PET/CT in suspected recurrence of breast cancer. Cancer 107:2545-2551

28. Veit-Haibach P, Antoch G, Beyer T et al (2007) FDG-PET/CT in restaging of patients with recurrent breast cancer: possible impact on staging and therapy. Br J Radiol 80:508-515

29. Kumar A, Kumar R, Seenu V et al (2009) The role of 18 F-FDG $\mathrm{PET} / \mathrm{CT}$ in evaluation of early response to neoadjuvant chemotherapy in patients with locally advanced breast cancer. Eur Radiol 19:1347-1357

30. Schiepers C (2003) PET/CT in Colorectal Cancer. J Nucl Med 44:1804-1805

31. Kinner S, Antoch G, Bockisch A et al (2007) Whole-body PET/ CT-colonography: a possible new concept for colorectal cancer staging. Abdom Imaging 32:606-612

32. Schmidt GP, Baur-Melnyk A, Haug A et al (2009) Whole-body MRI at $1.5 \mathrm{~T}$ and $3 \mathrm{~T}$ compared with FDG-PET-CT for the detection of tumour recurrence in patients with colorectal cancer. Eur Radiol 19:1366-1378

33. Schaefer O, Langer M (2007) Detection of recurrent rectal cancer with CT, MRI and PET/CT. Eur Radiol 17:2044-2054

34. Al-Ibraheem A, Buck A, Krause BJ et al (2009) Clinical Applications of FDG PET and PET/CT in Head and Neck Cancer. J Oncol 2009:208725

35. Hustinx R, Lucignani G (2010) PET/CT in head and neck cancer: an update. Eur J Nucl Med Mol Imaging 37:645-651

36. Yoon DY, Hwang HS, Chang SK et al (2009) CT, MR, US, 18FFDG PET/CT, and their combined use for the assessment of cervical lymph node metastases in squamous cell carcinoma of the head and neck. Eur Radiol 19:634-642

37. Kitajima K, Murakami K, Yamasaki E et al (2008) Performance of FDG-PET/CT for diagnosis of recurrent uterine cervical cancer. Eur Radiol 18:2040-2047

38. Grigsby PW (2008) The prognostic value of PET and PET/CT in cervical cancer. Cancer Imaging 8:146-155

39. Kidd EA, Siegel BA, Dehdashti F et al (2010) Lymph node staging by positron emission tomography in cervical cancer: relationship to prognosis. J Clin Oncol 28:2108-2113

40. Ng SH, Chan SC, Yen TC et al (2010) Comprehensive imaging of residual/ recurrent nasopharyngeal carcinoma using whole-body MRI at 3 T compared with FDG-PET-CT. Eur Radiol 20:22292240 
41. Freudenberg LS, Frilling A, Kuhl $\mathrm{H}$ et al (2007) Dual-modality FDG-PET/CT in follow-up of patients with recurrent iodinenegative differentiated thyroid cancer. Eur Radiol 17:3139-3147

42. Freudenberg LS, Antoch G, Jentzen W et al (2004) Value of (124) I-PET/CT in staging of patients with differentiated thyroid cancer. Eur Radiol 14:2092-2098

43. Aukema TS, Valdes Olmos RA, Wouters MW et al (2010) Utility of preoperative 18F-FDG PET/CT and brain MRI in melanoma patients with palpable lymph node metastases. Ann Surg Oncol 17:2773-2778

44. Schoder H, Larson SM, Yeung HW (2004) PET/CT in oncology: integration into clinical management of lymphoma, melanoma, and gastrointestinal malignancies. J Nucl Med 45(Suppl 1):72S$81 \mathrm{~S}$

45. Strobel K, Dummer R, Husarik DB et al (2007) High-risk melanoma: accuracy of FDG PET/CT with added CT morphologic information for detection of metastases. Radiology 244:566-574

46. Allen-Auerbach M, de Vos S, Czernin J (2008) The impact of fluorodeoxyglucose-positron emission tomography in primary staging and patient management in lymphoma patients. Radiol Clin North Am 46:199-211, vii

47. Hofman MS, Smeeton NC, Rankin SC et al (2009) Observer variation in interpreting $18 \mathrm{~F}-\mathrm{FDG}$ PET/CT findings for lymphoma staging. J Nucl Med 50:1594-1597

48. Kasamon YL, Jones RJ, Wahl RL (2007) Integrating PET and $\mathrm{PET} / \mathrm{CT}$ into the risk-adapted therapy of lymphoma. J Nucl Med 48(Suppl 1):19S-27S

49. Kwee TC, Kwee RM (2009) Combined FDG-PET/CT for the detection of unknown primary tumors: systematic review and meta-analysis. Eur Radiol 19:731-744

50. Rosenbaum SJ, Lind T, Antoch G et al (2006) False-positive FDG PET uptake - the role of PET/CT. Eur Radiol 16:1054-1065

51. Costelloe CM, Murphy WA Jr, Chasen BA (2009) Musculoskeletal pitfalls in 18F-FDG PET/CT: pictorial review. AJR Am J Roentgenol 193:WS1-WS13, Quiz S26-30

52. Adams MC, Turkington TG, Wilson JM et al (2010) A systematic review of the factors affecting accuracy of SUV measurements. AJR Am J Roentgenol 195:310-320

53. Schiepers C, Hoh CK, Nuyts J et al (2008) 1-11C-acetate kinetics of prostate cancer. J Nucl Med 49:206-215

54. Apostolova I, Wiemker R, Paulus T et al (2010) Combined correction of recovery effect and motion blur for SUV quantification of solitary pulmonary nodules in FDG PET/CT. Eur Radiol $20: 1868-1877$
55. Blake MA, Setty BN, Cronin CG et al (2010) Evaluation of the effects of oral water and low-density barium sulphate suspension on bowel appearance on FDG-PET/CT. Eur Radiol 20:157-164

56. de Geus-Oei LF, Vriens D, van Laarhoven HW et al (2009) Monitoring and predicting response to therapy with $18 \mathrm{~F}-\mathrm{FDG}$ PET in colorectal cancer: a systematic review. J Nucl Med 50 (Suppl 1):43S-54S

57. Jarritt PH, Carson KJ, Hounsell AR et al (2006) The role of PET/ CT scanning in radiotherapy planning. Br J Radiol $79 \mathrm{Spec}$ No 1: S27-35

58. Ford EC, Herman J, Yorke E et al (2009) 18F-FDG PET/CT for image-guided and intensity-modulated radiotherapy. J Nucl Med 50:1655-1665

59. Messa C, Di Muzio N, Picchio M et al (2006) PET/CT and radiotherapy. Q J Nucl Med Mol Imaging 50:4-14

60. Minn H, Suilamo S, Seppala J (2010) Impact of PET/CT on planning of radiotherapy in head and neck cancer. Q J Nucl Med Mol Imaging 54:521-532

61. Budinger TF (1983) Time-of-flight positron emission tomography: status relative to conventional PET. J Nucl Med 24:73-78

62. Allemand R, Gresset C, Vacher J (1980) Potential advantages of a cesium fluoride scintillator for a time-of-flight positron camera. $\mathrm{J}$ Nucl Med 21:153-155

63. Judenhofer MS, Wehrl HF, Newport DF et al (2008) Simultaneous PET-MRI: a new approach for functional and morphological imaging. Nat Med 14:459-465

64. Pichler BJ, Judenhofer MS, Catana C et al (2006) Performance test of an LSO-APD detector in a 7-T MRI scanner for simultaneous PET/MRI. J Nucl Med 47:639-647

65. Pichler BJ, Judenhofer MS, Wehrl HF (2008) PET/MRI hybrid imaging: devices and initial results. Eur Radiol 18:1077-1086

66. Boss A, Bisdas S, Kolb A et al (2010) Hybrid PET/MRI of intracranial masses: initial experiences and comparison to PET/ CT. J Nucl Med 51:1198-1205

67. Fischer MA, Nanz D, Hany T et al (2010) Diagnostic accuracy of whole-body MRI/DWI image fusion for detection of malignant tumours: a comparison with PET/CT. Eur Radiol. doi:10.1007/ s00330-010-1929-x

68. Hofmann M, Steinke F, Scheel V et al (2008) MRI-based attenuation correction for PET/MRI: a novel approach combining pattern recognition and atlas registration. J Nucl Med 49:1875-1883

69. Phelps ME (2000) Inaugural article: positron emission tomography provides molecular imaging of biological processes. Proc Natl Acad Sci U S A 97:9226-9233 